\title{
EDQM-HC Administration Device Terminology
}

National Cancer Institute

\section{Source}

National Cancer Institute. EDQM-HC Administration Device Terminology. NCI Thesaurus.

Code C150110.

Terminology subset about devices that are used to administer a pharmaceutical product. 